increases with an increase in conductivity. Based on the findings, the researchers anticipate that a further improvement in the photocathode quantum efficiency can be achieved with higher conductivity of the material. Although a $30 \% \mathrm{QE}$ is still lower than QEs reported in the literature for the deep-UV (near $280 \mathrm{~nm}$ and below), the promise of achieving higher responsivity in a photocathode-type detector exists. Also, the photocathode tubes showed high stability over time.

The researchers are now working to improve the conductivity of this material to increase its efficiency as well as to improve the solar blindness of the resulting detectors.

"By adding electrically active impurities to gallium nitride, we have improved the optical properties of the material, making it very sensitive to ultraviolet light, but we know we can do even better," said Wessels. "Our goal is to make the device with greater than $50 \%$ quantum efficiency, which means it will be 10 times better than the detectors used in the Hubble Space Telescope." Quantum efficiencies as high as $90 \%$ are theoretically possible, according to the research team.

SHIMING WU

\section{Polymeric Material Self-Repairs Without Addition of Chemicals}

While many plastic materials are strong and resistant to breaking, once fractured, the broken pieces are cracked irreversibly. Although progress has been made in the development of self-healing polymers with the use of polymer catalysts or glues, further questions still remain concerning the long-term stability of the catalyst and the ability of the material to self-heal multiple times. Fred Wudl, professor of organic chemistry at the University of California-Los Angeles and director of UCLA's Exotic Materials Institute, led a research group of chemists and engineers who developed a transparent plastic that, when fractured, mends itself with heating.

Unlike other self-healing plastics developed previously, this material, called automend, repairs itself without the addition of a catalyst or other chemicals. As reported in the March 1 issue of Science, automend is an extremely hard and fully transparent polymer material prepared by the Diels-Alder cycloaddition of a multi-diene and a multi-dienophile. Its density is $1.37 \mathrm{~g} / \mathrm{cm}^{3}$ with an index of refraction of 1.56 at $24^{\circ} \mathrm{C}$, its Young's modulus is $4.72 \mathrm{GPa}$, and its Poisson ratio is 0.349 . Its mechanical properties are similar to commercial epoxy resins.

Automend can be fractured and healed multiple times through heating at $120^{\circ} \mathrm{C}$, due to reversible cross-linking covalent bonds formed between the polymer chains and branches. While being heated, these bonds between the polymer chains are broken; but during cooling, the polymer will be remanufactured by reforming those broken bonds. Solid-state nuclear magnetic resonance spectroscopy showed that when the temperature is above $120^{\circ} \mathrm{C}, \sim 30 \%$ of intermonomer linkages disconnect and then reconnect upon cooling. After healing, the original fracture is invisible, according to scanning electron microscope images, and the polymer can retain $\sim 60 \%$ of its original fracture strength from the fracture tests.

Although this new polymeric structuralengineering material has potential use in large lenses and housings for radar or communications equipment, the researchers addressed some future studies for improvement such as the maleimide monomer's high melting temperature, curing time of the material, and service temperature range.

YuE Hu

\section{Transport Properties Measured by Hybrid Molecular-Assembly Approach}

Progress in the development of nanoscale molecular electronics requires the rapid screening of molecular systems for their electronic-transport properties. An essential element of this process involves the connection of electrodes to the molecules of interest. A team of researchers led by Raymond K. Tsui in the Physical Sciences Research Laboratories at Motorola Laboratories in Tempe, Ariz., has devised a hybrid molecular-assembly technique to facilitate the rapid screening of candidate molecules: first, forming a self-assembled monolayer (SAM) on a pair of prepatterned electrodes, then using metallic nanoparticles for gap-bridging under an ac field as an alternative fabrication approach to provide a solution for transport measurements of molecules.

As reported in the April 15 issue of Applied Physics Letters, the researchers fabricated gold nanoparticle electrodes using a combination of photolithography and electron-beam lithography on an oxidized Si substrate. With the lift-off technique, fine features of the electrodes were patterned by a poly(methyl methyacrylate) (PMMA) resist process. After the substrate was cleaned to remove oxidized $\mathrm{Au}$, the researchers assembled nanoparticles by applying an ac bias (0.5-2.5 V, peak-to-peak) ranging from $1 \mathrm{MHz}$ to $10 \mathrm{MHz}$ for 5-20 s. By applying an ac field, a dielectrophoretic force was creat- ed that pulled the nanoparticles in the direction of maximum field strength between the gap. The researchers measured various sizes of $\mathrm{Au}$ nanoparticles, ranging from $40 \mathrm{~nm}$ to $100 \mathrm{~nm}$, trapped between the gap with a yield of almost $100 \%$. They also discovered that as soon as the gold particle bridged the gap, the electric field in the gap was reduced, which tended to prevent additional accumulation. Additionally, since the resistance of the $\mathrm{Au}$ electrode-particle assembly varied from $100 \mathrm{k} \Omega$ to a few tens of megaohms, a larger number of nanoparticles could be clustered in the gap region if the nanoparticle size was smaller than the gap.

The researchers selected 2,5-diphenylethynyl-4',4"-dithioacetyl-nitrobenzene $\left(1^{\prime}\right)$ as a test molecule. The SAM tested consisted of a Au-1'-Au-1"-Au structure and showed qualitative current-voltage $(I-V)$ measurements with two negative differential resistance peaks similar to those observed in semiconductor heterostructures.

"Our approach is relatively simple and... can potentially allow a quick and easy way to characterize a large number of electronic molecules," said Islamshah Amlani, a senior engineer at Motorola Labs.

KINSON C. KAM

\section{Field-Emission Current Induces High Stable Temperature in Multiwalled Carbon Nanotubes}

S.T. Purcell and colleagues at the Université Claude Bernard Lyon 1 have induced stable temperatures of up to $2000 \mathrm{~K}$ in multiwalled carbon nanotubes (MWNTs) by field-emission current. As reported in the March 11 issue of Physical Review Letters, the researchers determined the temperature and resistance of individual MWNTs with field-emission electron spectroscopy. Both the high temperature and the light emission seen during field emission come from Joule heating of the MWNTs. The researchers propose that field emission can be used to improve the structure by heat treating. They also determined that the resistance of the MWNTs decreases with temperature.

The MWNTs were grown by chemical vapor deposition onto Ni tips. Scanning electron microscopy showed that the MWNTs were straight, $\sim 30 \mathrm{~nm}$ in diameter, and $\sim 40 \mu \mathrm{m}$ long. Transmission electron microscopy confirmed that they were multiwalled.

Field-emission experiments were conducted in ultrahigh vacuum of $\sim 7 \times 10^{-11}$ Torr. The total-energy distributions (TEDs) of the emitted electrons were measured with a hemispherical electronenergy analyzer. Although many MWNTs 
grew on each $\mathrm{Ni}$ tip, the field-emission measurements were specific to individual nanotubes for two reasons. First, the emission current $\left(I_{\mathrm{FE}}\right)$ is an exponential function of field, so only a few MWNTs are able to emit. Second, the geometry of field emission allows the contributions of each MWNT to the field-emission pattern to be easily distinguished.

The researchers determined the temperature and resistance from TEDs obtained for various $I_{\mathrm{FE}}$. As $I_{\mathrm{FE}}$ increased, the TEDs became broader and shifted to lower energy. The broadening is partly explained by the rise in the temperature due to Joule heating along the MWNTs. The shift to lower energy results from an IR drop along the tube.

\section{ELIZABETH SHACK}

\section{Carbon Nanotube Bandgaps Manipulated with Metallofullerenes}

A team of researchers from Seoul National University, Soongsil University, and Nagoya University has detected localized bandgap-energy changes in singlewalled carbon nanotubes (SWNTs) that contain fullerene $\left(\mathrm{C}_{82}\right)$ encapsulated gadolinium ions (GdMF). As reported in the February 28 issue of Nature, research led by Young Kuk from Seoul National University demonstrated that bandgap energies of single SWNTs were tailored with $~ 3-\mathrm{nm}$ accuracy using GdMF insertion to induce changes in nanotube structure and electronic environment and divide the nanotube into multiple quantum dots. Scanning tunneling microscopy (STM) was employed to monitor changes in tunneling conductivity to probe for breaks in symmetry imposed on the SWNTs. The major motivation is the development of nanoscale electronic devices as alternatives to Si-based devices.

The metallofullerene insertion into SWNTs was accomplished by heating the two in a glass ampoule at $500^{\circ} \mathrm{C}$ for three days. The diameters of the GdMF inserted were slightly larger than the SWNTs and could be spaced in a systematic fashion (every $1.1 \mathrm{~nm}$ ) or quasi-periodically (every 1-3 nm), depending upon either a sonication or annealing method. The GdMF and SWNTs were both fabricated by a dc arcdischarge method, with the GdMF purified through high-performance liquid chromatography, and SWNTs purified by treatment with acid.

Using STM operated at $\sim 5 \mathrm{~K}, \mathrm{~d} I / \mathrm{d} V$ spectra were obtained from the images to estimate the bandgap at 512 points along a 10-nm section of a single SWNT. In areas that did not contain GdMF density, the SWNTs had a bandgap of $0.43 \mathrm{eV}$, while in the areas of GdMF insertion, the bandgap decreased to $0.17 \mathrm{eV}$. The evidence for such changes in tunneling conductivity along single SWNTs could be easily seen in the STM images, with bright "spots" appearing in a periodic fashion. Such bright spots are likely to be from both bandgap change and also topography change. The researchers believe insertion of GdMF into SWNTs has two effects along this line. First, the electron transfer from both the $\mathrm{Au}(111)$ substrate and the GdMF cluster may lead to charge transfers, resulting in the bandgap change. Second, the insertion of a GdMF with a greater diameter than the SWNTs introduces an elastic strain of the SWNTs, contributing to additional bandgap change.

The researchers believe that this ability to transform a SWNT into a one-dimensional multiple-quantum-dot system may contribute to the fields of nanoelectronics, nanooptoelectronics, and perhaps even a quantum cascade laser or quantum computer. MATHEW M. MAYE

\section{Computational Technique Facilitates Modeling of Fluid Transport in Porous Media}

Computational materials scientist Clint Van Siclen, from the Idaho National Engineering and Environmental Laboratory, has demonstrated a theoretical approach to modeling fluid transport in porous, variable materials such as rock. Through his approach, called the walker diffusion method (WDM), Van Siclen calculates how electricity "diffuses" through a composite material. The WDM is based on the concept of a single random walker-a theoretical construct that "walks" through the material, randomly taking a step in one direction, a step in another direction, and so on. Left alone long enough, the walker will eventually explore all the potential paths available. By tracking these paths, Van Siclen is able to map out the fluid-flow routes in a permeable material, including sharp twists and turns or the tiniest of crack lines.

As reported in the February issue of Physical Review E, Van Siclen maps out the movements of the walker by first digitizing the structure of the porous material. That digitized representation is thus a square or cubic array of pixels or voxels, each of which is open or closed, corresponding to pore space and impermeable rock, respectively. If a pixel is open, there is a high probability that a walker will travel through that space. If the pixel is closed, then a walker will not be able to occupy that space. In a relatively short period of time, a walker can explore the accessible space using these simple, quickly comput- ed probabilities. The calculations go even faster if several non-interacting walkers are used. These paths reveal the overall physical structure of the material.

With the conventional approach to calculating flow paths, called the finitedifference method (FDM), researchers take the digitized sample and construct a very large set of finite-difference equations, that is, equations that define the difference between the values of a function at two discrete points. Those equations have to be solved simultaneously, a task that strains the capabilities of all but the largest computers for realistically sized systems. In contrast, the WDM can be performed on a typical PC.

Furthermore, Van Siclen reports, the WDM obtains the "correlation length" for the material under study. This parameter is the size above which a specimen is uniform (homogeneous) with respect to the transport property of interest, such as fluid permeability, and below which it is variable (heterogeneous). The existence of the correlation length, which may be tens or hundreds of meters in the case of fractured bedrock, thus fundamentally limits the extent to which results from laboratory experiments are applicable to field sites.

According to Van Siclen, the WDM enables very large, or highly resolved,

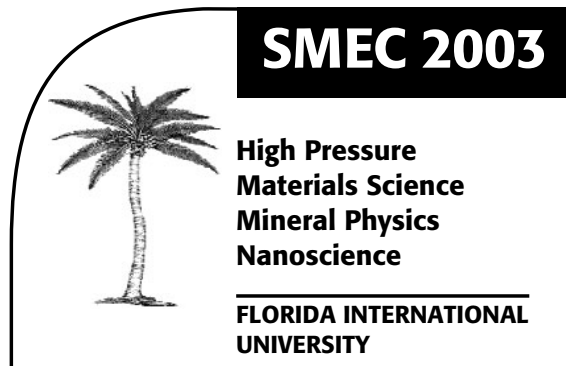

\section{First Announcement}

CeSMEC will host the second meeting of the Study of Matter at Extreme Conditions (SMEC) 24-27 March of 2003. The focus of the 4-day meeting will be to promote the integration of mineral-physics, high-pressure chemistry/ physics and materials science (including nanomaterials). Sponsored by the Florida International University Division of Sponsored Research and Colleges of Arts \& Sciences and Engineering, the meeting will bring together scholars from all over the world at FIU's Biscayne Bay Campus-proximal to Miami Beach, the Everglades, and the Florida Keys.

Interested scientists are urged to email: saxenas@fiu.edu.

We welcome your input concerning specific topics/issues suitable for a session or forum.

Circle No. 5 on Inside Back Cover 